\section{Fisch ist nicht gleich Fisch}

$\mathrm{N}$ icht nur Gourmets werden bestätigen: Fisch ist nicht gleich Fisch. Auch Allergiekranke machen die Erfahrung, dass sie auf verschiedene Spezies und unterschiedliche Zubereitungen mehr oder weniger stark reagieren. Möglicherweise könnte sich dies durch eine variable Expression des Majorallergens Parvalbumin erklären lassen.

Diese Hypothese überprüfte eine Arbeitsgruppe um Dr. Annette Kühn, Luxemburg: Die Wissenschaftler nahmen zunächst eine Einschätzung des Gehalts an Parvalbumin in rohem Muskelfleisch verschiedener Fischsorten mittels SDSPAGE vor. Bei Proben aus dem Muskelfleisch der Makrele betrug das Parvalbumin demnach weniger als $0,5 \mathrm{mg}$ pro Gramm Gewebe, bei Lachs, Forelle, Dorsch und Rotbarsch lag der Parvalbumin-Gehalt zwischen 1 und $3 \mathrm{mg} / \mathrm{g}$ und bei Karpfen und Hering bei mehr als $3 \mathrm{mg} / \mathrm{g}$.

Mittels spezifischem ELISA wurde anhand polyklonaler Maus-Antiseren eine genauere Quantifizierung des Parvalbumin-Gehalts vorgenommen: Thunfisch enthielt demnach unter 0,05 mg Parvalbumin pro g Muskelfleisch, Makrele 0,3$0,7 \mathrm{mg} / \mathrm{g}$, Dorsch 1,5-2,5 mg/g, Lachs 1,9-2,5 mg/g, Forelle $2-2,5 \mathrm{mg} / \mathrm{g}$, Rotbarsch $2-3 \mathrm{mg} / \mathrm{g}$, Karpfen $2,5-5 \mathrm{mg} / \mathrm{g}$ und Hering 3,8-5,7 mg/g. In gekochtem, geräuchertem oder eingelegtem Fisch lag die Konzentration des Allergens um 20$60 \%$ niedriger als in rohem Fisch.

Der Gehalt an Parvalbumin kann sich diesen Befunden zufolge bei häufig konsumierten Fischsorten um das 100-fache

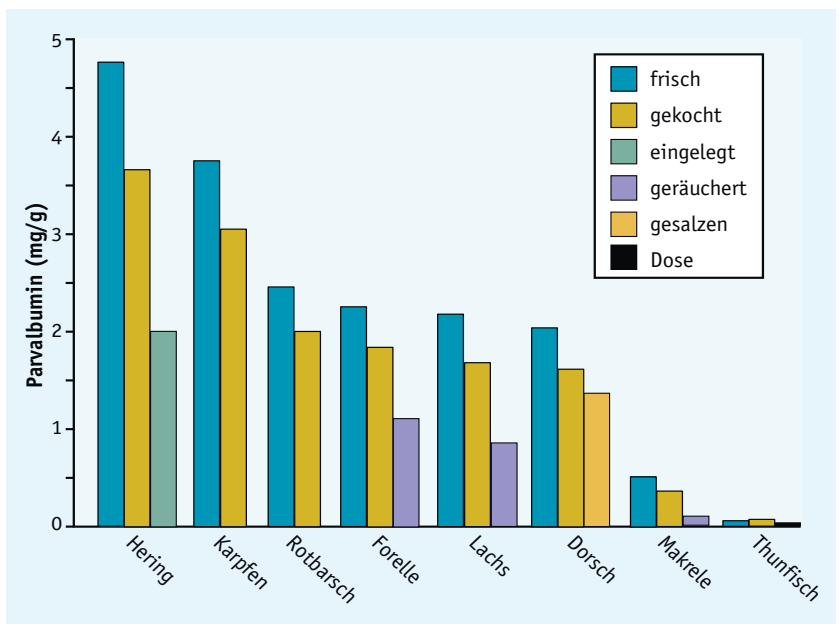

Gehalt an Parvalbumin in Abhängigkeit der Fischspezies und der Zubereitungsform

unterscheiden und damit wesentlich zur variablen Allergenität verschiedener Spezies und Zubereitungen beitragen, schlussfolgerte Kühn. Da jedoch auch Fischsorten wie Thunfisch, die wenig Parvalbumin enthalten, in einzelnen Fällen Allergien auslösen können, scheinen auch weitere Allergene eine wichtige Rolle zu spielen.

abd

Kuehn A, Scheuermann T, Hilger C, Hentges F. Quantification of parvalbumin in fresh and processed fish

\section{Kautest bei Nahrungsmittelallergie}

S ubjektive Beschwerden wie Juckreiz und Kribbeln an der Mundschleimhaut sind charakteristisch für das orale Allergiesyndrom. Objektive Symptome wie ein sichtbares Anschwellen der Mundschleimhaut sind dagegen selten. Eine Arbeitsgruppe um Tanja Friedl, München, entwickelte nun eine standardisierte Testmethode, um ein orales Allergiesyndrom zu objektivieren.

Der epimuköse enorale Provokationstest (EEP) basiert auf der Messung von Mastzelltryptase im Speichel. Hierfür wird zunächst eine Watterolle für eine Minute unter die Zunge des Patienten gelegt, um eine Speichelprobe für die Ermittlung des Mastzelltryptase-Basiswerts zu gewinnen. Anschließend kaut der Patient definierte Mengen des verdächtigen Nahrungsmittels, bis subjektive Symptome auftreten, und spuckt dann die Speise aus. Danach werden
Speichelproben für die Bestimmung der Mastzelltryptase-Konzentration entnommen.

Bislang liegen die Ergebnisse von 18 solcher Provokationstestungen bei Patienten mit oralem Allergiesyndrom bzw. Nahrungsmittelanaphylaxie vor, bei denen eine Sensibilisierung durch einen positiven Pricktest und/oder spezifische IgE-Antikörper im Serum nachgewiesen worden war: Bei über $80 \%$ der Testungen wurde ein Anstieg der Mastzelltryptase im Speichel ermittelt. Die maximale Konzentration wurde meist nach vier Minuten gemessen. In der klinischen Praxis sollte die Messung daher zu diesem Zeitpunkt erfolgen. Kontrolltests mit vertragenen Nahrungsmitteln führten zu keinem Anstieg der Mastzelltryptase im Speichel. Bei zwei Probanden mit bekannter Anaphylaxie kam es nach längerem Kauen des auslösen- den Nahrungsmittels erstmals zu den Symptomen eines oralen Allergiesyndroms, die mit erhöhter Mastzelltryptase im Speichel einhergingen.

Die Messung der MastzelltryptaseKonzentration im Speichel erlaube es somit, ein orales Allergiesyndrom zu objektivieren, resümierte Tanja Friedl. Der epimuköse enorale Provokationstest könne möglicherweise auch manchem Patienten mit Nahrungsmittelanaphylaxie eine orale systemische Provokation ersparen.

Bei der epimukösen enoralen Provokationstestung kann es jedoch auch zu systemischen Reaktionen kommen, wie Dr. Annett Walker, München, am Beispiel eines Patienten mit einer durch Sesam ausgelösten Anaphylaxie veranschaulichte: Bei dem 33-jährigen Mann war es in der Vorgeschichte mehrfach rund 20 Minuten nach Verzehr sesamhaltiger „Mehrkornsemmeln“ zu Quincke-Ödemen, generalisierter Urtikaria und Atemnot gekommen. 